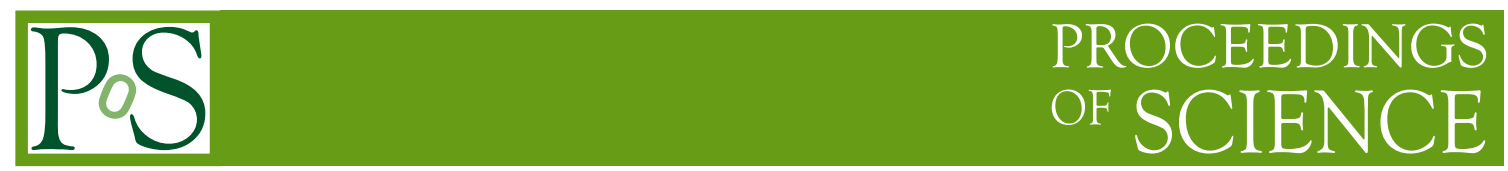

\title{
Performance studies of the Belle II Silicon Vertex Detector with data taken at the DESY test beam in April 2016
}

T. Lück ${ }^{h, i}$, K. Adamczyk ${ }^{s}$, H. Aihara ${ }^{o}$, C. Angelini ${ }^{h, i}$, T. Aziz $^{g}$, V. Babu $^{g}$, S. Bacher ${ }^{s}$, S. Bahinipati ${ }^{d}$, E. Barberio ${ }^{a}$, Ti. Baroncelli ${ }^{a}$, To. Baroncelli ${ }^{a}$, A. K. Basith ${ }^{e}$, G. Batignani $^{h, i}$, A. Bauer ${ }^{b}$, P. K. Behera ${ }^{e}$, T. Bergauer ${ }^{b}$, S. Bettarini ${ }^{h, i}$, B. Bhuyan ${ }^{f}$, T. Bilka ${ }^{c}$, F. Bosi $^{i}$, L. Bosisio ${ }^{j, k}$, A. Bozek ${ }^{s}$, F. Buchsteiner ${ }^{b}$, L. Bulla ${ }^{b}$, G. Caria ${ }^{a}$, G. Casarosa ${ }^{i}$, M. Ceccanti $^{i}$, D. Červenkov ${ }^{c}$, S. R. Chendvankar ${ }^{g}$, N. Dash ${ }^{d}$, G. De Pietro $^{h, i}$, S. T. Divekar ${ }^{g}$, Z. Doležal ${ }^{c}$, D. Dutta ${ }^{g}$, F. Forti $^{h, i}$, M. Friedl ${ }^{b}$, K. Hara ${ }^{p}$, T. Higuchi ${ }^{l}$, T. Horiguchi ${ }^{n}$, C. Irmler ${ }^{b}$, A. Ishikawa ${ }^{n}$, H. B. Jeon ${ }^{q}$, C. Joo ${ }^{l}$, J. Kandra $^{c}$, N. Kambara ${ }^{p}$, K. H. Kang ${ }^{q}$, T. Kawasaki ${ }^{m, C}$, P. Kodyš ${ }^{c}$, T. Kohriki ${ }^{p}$, S. Koike ${ }^{p, D}$, M. M. Kolwalkar ${ }^{g}$, R. Kumar ${ }^{t}$, W. Kun ${ }^{o}$, P. Kvasnička ${ }^{c}$, C. La Licata ${ }^{j, k}$, L. Lanceri ${ }^{j, k}$, J. Lettenbichler ${ }^{b}$, J. Libby ${ }^{e}$, M. Maki ${ }^{p}$, P. Mammini ${ }^{i}$, A. Martini ${ }^{h, i}$, S. N. Mayekar ${ }^{g}$, G. B. Mohanty ${ }^{g}$, S. Mohanty ${ }^{g, A}$, T. Morii ${ }^{l}$, K. R. Nakamura ${ }^{p}$, Z. Natkaniec ${ }^{s}$, Y. Onuki ${ }^{o}$, W. Ostrowicz ${ }^{s}$, A. Paladino ${ }^{h, i}$, E. Paoloni ${ }^{h, i}$, H. Park ${ }^{q}$, F. Pilo ${ }^{i}$, A. Profeti ${ }^{i}$, K. K. Rao ${ }^{g}$, I. Rashevskaya ${ }^{k, B}$, G. Rizzo ${ }^{h, i}$, Resmi P. K. ${ }^{e}$, M. Rozanska ${ }^{s}$, J. Sasaki ${ }^{o}$, N. Sato ${ }^{p}$, S. Schultschik ${ }^{b}$, C. Schwanda ${ }^{b}$, B. Schwenker ${ }^{u}$, Y. Seino $^{m}$, N. Shimizu ${ }^{o}$, U. Stolzenberg $^{u}$, J. Stypula ${ }^{s}$, J. Suzuki ${ }^{p}$, S. Tanaka ${ }^{p}$, G. N. Taylor ${ }^{a}$, R. Thalmeier ${ }^{b}$, R. Thomas ${ }^{g}$, T. Tsuboyama ${ }^{p}$, S. Uozumi ${ }^{q}$, P. Urquijo ${ }^{a}$, L. Vitale ${ }^{j, k}$, M. Watanabe ${ }^{l}$, S. Watanuki ${ }^{n}$, I. J. Watson ${ }^{o}$, J. Webb $^{a}$, J. Wiechczynski ${ }^{s}$, S. Williams ${ }^{a}$, B. Würkner ${ }^{b}$, H. Yamamoto ${ }^{n}$, H. Yin ${ }^{b}$, T. Yoshinobu ${ }^{p}$, L. Zani ${ }^{h, i}$, (Belle-II SVD Collaboration) 
School of Physics, University of Melbourne, Melbourne, Victoria 3010, Australia

${ }^{b}$ Institute of High Energy Physics, Austrian Academy of Sciences, 1050 Vienna, Austria

${ }^{c}$ Faculty of Mathematics and Physics, Charles University, 12116 Prague, Czech Republic

" Georg-August-Universität Göttingen, 37073 Göttingen, Germany

${ }^{d}$ Indian Institute of Technology Bhubaneswar, Satya Nagar, India

${ }^{e}$ Indian Institute of Technology Madras, Chennai 600036, India

${ }^{f}$ Indian Institute of Technology Guwahati, Assam 781039, India

${ }^{g}$ Tata Institute of Fundamental Research, Mumbai 400005, India, ${ }^{A}$ also at Utkal University, Bhubaneswar 751004, India

${ }^{h}$ Dipartimento di Fisica, Università di Pisa, I-56127 Pisa, Italy

${ }^{i}$ INFN Sezione di Pisa, I-56127 Pisa, Italy

${ }^{j}$ Dipartimento di Fisica, Università di Trieste, I-34127 Trieste, Italy

${ }^{k}$ INFN Sezione di Trieste, I-34127 Trieste, Italy, ${ }^{B}$ presently at TIFPA - INFN, I-38123 Trento, Italy

${ }^{l}$ Kavli Institute for the Physics and Mathematics of the Universe (WPI), University of Tokyo, Kashiwa 277-8583, Japan

${ }^{m}$ Department of Physics, Niigata University, Niigata 950-2181, Japan, ${ }^{C}$ presently at Kitasato University, Sagamihara 252-0373, Japan

${ }^{n}$ Department of Physics, Tohoku University, Sendai 980-8578, Japan

${ }^{\circ}$ Department of Physics, University of Tokyo, Tokyo 113-0033, Japan

${ }^{p}$ High Energy Accelerator Research Organization (KEK), Tsukuba 305-0801, Japan, ${ }^{D}$ deceased

${ }^{q}$ Department of Physics, Kyungpook National University, Daegu 702-701, Korea

${ }^{s}$ H. Niewodniczanski Institute of Nuclear Physics, Krakow 31-342, Poland

${ }^{t}$ Punjab Agricultural University, Ludhiana 141004, India

Belle II is a multipurpose detector currently under construction and it will be operated at the next generation B-factory SuberKEKB in Japan. The main Belle II devices, devoted to the vertex reconstruction, are the Silicon Vertex Detector (SVD) and the Pixel Detector (PXD). In April 2016 a sector of the Belle II SVD and PXD has been tested in a beam of high energetic electrons at the test beam facility at DESY Hamburg (Germany). We report here the results for the hit efficiency estimation and the measurement of the resolution for the Belle II silicon vertex detector. Hit efficiencies are measured to be on average above $99.5 \%$ and the measured resolution is within the expectations.

The 25th International workshop on vertex detectors September 26-30, 2016

La Biodola, Isola d'Elba, ITALY

${ }^{*}$ Speaker. 


\section{Introduction}

The Belle II detector [1] is a multipurpose detector which will be operated at the asymmetric $B$-Factory SuperKEKB [2] (Japan). Measurements planned to perform at the Belle II experiment aim at testing the standard model of particle physics with high precision. An unprecedented instantaneous luminosity of up to $8 \times 10^{35} \mathrm{~cm}^{-2} \mathrm{~s}^{-1}$ will be provided by the accelerator which poses extreme requirements on the sub-detectors of Belle II. The reconstruction of charged particle positions close to the interaction point is provided by the Vertex Detector (VXD), composed of the Pixel Detector (PXD) [1, 3], made of two layers of sensors based on DEPFET pixels, and of the Silicon Vertex Detector (SVD) [1, 4], composed of four layers of double sided silicon strip detectors. Data taking of Belle II is foreseen to start in 2017, with first physics runs with the completed detector in 2018.

To achieve the physics goals of Belle II it is fundamental to reconstruct tracks with a high efficiency and a good resolution. Performance of prototypes of the PXD and SVD modules have been measured in previous testbeam campaigns. In April 2016 a full sector of the Vertex Detector, PXD and SVD, with its final configuration, has been tested with a beam of high energetic electrons at the test beam facility at DESY Hamburg. This was the first test of the SVD ladders in their final design which will be utilized in the Belle II experiment.

\section{The Silicon Vertex Detector}

The Silicon Vertex Detector (SVD) is one of the main track finding devices of the Belle II detector. It consists of 4 layers (L3, L4, L5, L6) of ladders made of several Double Sided Silicon Strip Detectors (DSSD) positioned at radii of $38 \mathrm{~mm}, 80 \mathrm{~mm}, 115 \mathrm{~mm}$, and $140 \mathrm{~mm}$ from the interaction point. The sensors have readout pitches in the $r-\phi$ direction (p-Side) between $50 \mu \mathrm{m}$ and 75 $\mu \mathrm{m}$, and between $160 \mu \mathrm{m}$ and $240 \mu \mathrm{m}$ in the $z$-direction (n-Side). To increase the resolution of the reconstructed position a float-

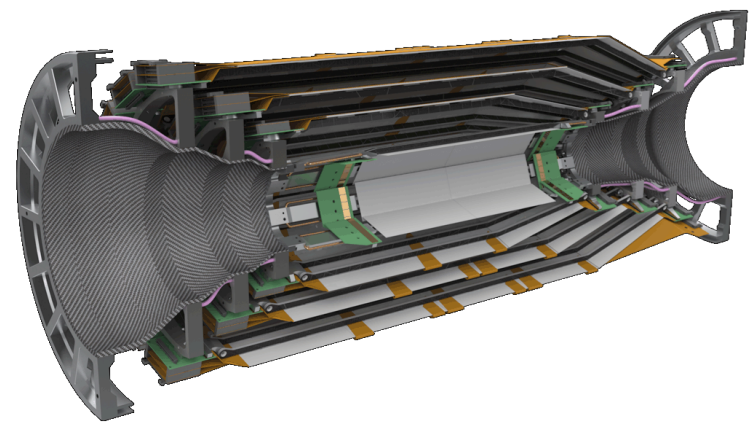

Figure 1: Shown is a 3D rendering of the Silicon Vertex Detector of the Belle II experiment. ing strip is implemented between two readout strips. The SVD features a fast readout by utilizing APV25 chips. An angle of $17^{\circ}<\theta<150^{\circ}$ is covered by the SVD, where $\theta$ is the angle as seen from the interaction point with respect to the $\mathrm{z}$-axis which is defined by the beam axis. Figure 1 shows a 3D rendering of the SVD. A detailed description of the SVD can be found in the references [1,4].

\section{Experimental setup}

The Vertex Detector sector tested with beams consist of 4 SVD ladders and 2 half ladders for the PXD. The setup was placed in a beam of electrons with energies of up to $5 \mathrm{GeV}$ which was 
provided by the DESY test beam facility. The beam hits the sensors at perpendicular incident angle. In addition a magnetic field of up to $1 \mathrm{~T}$ perpendicular to the beam was applied. The readout was performed by the full readout - chain foreseen to be used for Belle II data taking in a downscaled version [5]. Several runs at various beam energies and various magnetic field strengths have been recorded (distributed over 4 weeks) for offline analysis. In addition to the SVD and the PXD, 6 DATURA telescope planes [6] (3 upstream and 3 downstream) with Mimosa 26 pixel detectors provided position information.

Beam was seen on 5 of the 15 sensors composing the 4 different ladders. A picture of the SVD ladders under test and a schematic of the setup of all sensors used can be found in Figure 2.

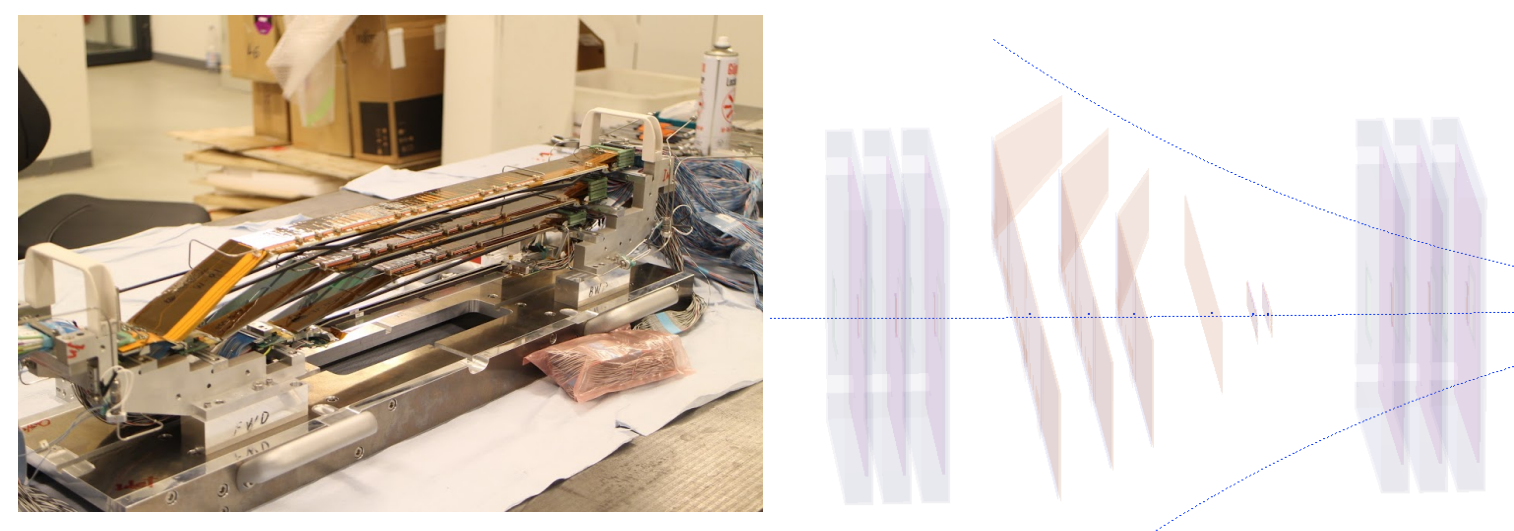

Figure 2: A picture of the four SVD ladders mounted on the supporting structure (Picture taken by Katsuro Nakamura (KEK)) is shown on the left-hand side. An event display for a typical test beam event to illustrate the arrangement of detectors is shown on the right-hand side. The detectors from left to right are three DATURA telescope planes, the four SVD ladders, two half ladders of PXD sensors and another three layers of DATURA telescope planes. The beam is going from the right to the left. The blue lines indicate true charged particle tracks (primary and secondary).

\section{Hit efficiency measurement}

The hit efficiency is measured only using three out of the four SVD layers of the full track finding procedure, where the reconstructed track is then used to predict the expected position on the fourth layer (the layer not used for the track reconstruction).

The track finding is performed by the track finding software implemented in the Belle II software framework [7]. The alignment of the sensors is done by the Belle II alignment framework utilizing the Millipede package. Track fits are performed by the Genfit2 package. For the analysis only events where a single track in the SVD has been reconstructed are used. To reject poorly reconstructed tracks we require that $P>0.02$, where $P$ is the p-value of the fit to the track. Only tracks from high momentum particles are considered for analysis by requiring that the reconstructed momentum is close to the nominal momentum expected from the beam $2<p_{f i t}<4 \mathrm{GeV} / \mathrm{c}$. In addition the uncertainty on the extrapolation of the reconstructed track on the sensor plane is required to be $<30-70 \mu \mathrm{m}$ (depending on layer and sensor side) to ensure a reasonable resolution for the predicted track position. 
The number of reconstructed hits is estimated by counting the number of events where a signal is found on a strip within $300 \mu \mathrm{m}$ of the predicted track position on the sensor under investigation. The efficiency is defined as $\varepsilon=$ \#hits is the number of events where a track fulfilling the afore mentioned conditions was found. The number of background hits, due to noisy strips or hits from secondary particles, for each event, is measured counting the number of hits for each strip which are further than $300 \mu \mathrm{m}$ away from the predicted track position. The probability of having a background hit on a strip, $P_{b k g}$, is typically below 0.001 , and it is subtracted from the efficiency $\varepsilon$. A systematic uncertainty on the estimate of the efficiency, equal to the probability of having background hits is conservatively assigned.

In Figure 3 we show the results for the efficiency as function of the strip number for a typical sensor. For all sensors under test we find an efficiency per strip which is on average above $99.5 \%$. The regions with low efficiency seen in Figure 3 are either already known defects of the sensor or are due to a gap between two sensors.
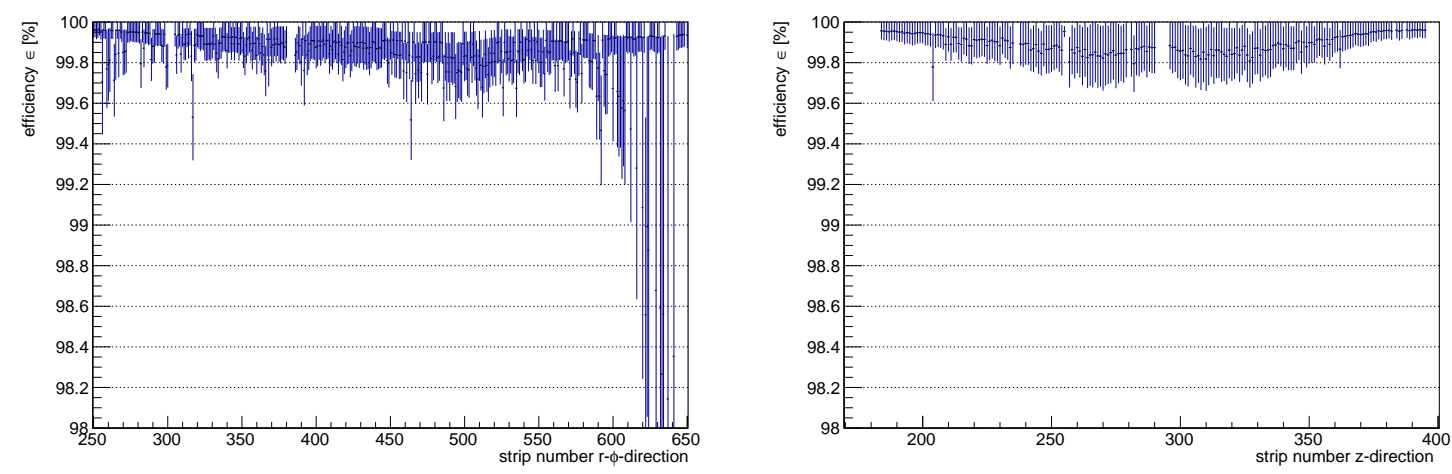

Figure 3: Efficiency as function of the strip number for the sensor on the 3rd ladder of the SVD sector under test (L5). On the left hand side the efficiency for the strips on the $r-\phi$ - direction are shown while on the right hand side the efficiencies for the $z$ - direction are shown. The error bars show the total uncertainty composed of statistical uncertainty and the systematic uncertainty due to background hits.

\section{Measurement of the hit resolution}

The intrinsic hit resolution is extracted from the distribution of the residuals, i.e. the distance between the reconstructed hit on the sensor and the extrapolated intersection of the reconstructed track with the sensor plane. Several terms contribute to the measured residual: hit resolution, uncertainty on the extrapolated track, multiple scattering. With the setup and beam energy used the multiple scattering contribution is expected to be negligible. To reduce the uncertainty due to the track extrapolation the high resolution telescope planes were also used in the analysis. As the telescope is not supported in the Belle II analysis and simulation framework (basf2), we use for alignment and track reconstruction a software developed to analyse test beam data [8,9]. This software is based on the iLC Soft software package [10]. A dataset of 539k events has been used for this analysis. The nominal beam momentum was set to $5 \mathrm{GeV}$ and the magnetic field was turned off. The track finding and fitting has been performed on all 12 planes ( 6 telescope planes +4 SVD planes +2 PXD planes). We require for each track to have a hit on at least 10 of the 12 sensor 


\begin{tabular}{c|c|c|c} 
ladder & $\begin{array}{c}\sigma-\phi \\
{[\mu \mathrm{m}]}\end{array}$ & $\begin{array}{c}\sqrt{\sigma^{2}-\sigma_{\text {track }}^{2}} \\
{[\mu \mathrm{m}]}\end{array}$ & $\begin{array}{c}p / 2 \sqrt{12} \\
{[\mu \mathrm{m}]}\end{array}$ \\
\hline L3 & $10.4 \pm 0.1$ & 8.2 & 7.2 \\
L4 & $11.7 \pm 0.1$ & 9.8 & 10.8 \\
L5 & $11.9 \pm 0.1$ & 10.0 & 10.8 \\
L6 & $11.4 \pm 0.1$ & 9.3 & 10.8
\end{tabular}

\begin{tabular}{c|c|c|c} 
ladder & $\begin{array}{c}\sigma \\
{[\mu \mathrm{m}]}\end{array}$ & $\begin{array}{c}\sqrt{\sigma^{2}-\sigma_{\text {track }}^{2}} \\
{[\mu \mathrm{m}]}\end{array}$ & $\begin{array}{c}p / 2 \sqrt{12} \\
{[\mu \mathrm{m}]}\end{array}$ \\
\hline L3 & $24.9 \pm 0.3$ & 23.6 & 23.1 \\
L4 & $35.5 \pm 0.4$ & 34.6 & 34.6 \\
L5 & $33.7 \pm 0.3$ & 32.7 & 34.6 \\
L6 & $31.4 \pm 0.3$ & 30.4 & 34.6
\end{tabular}

Table 1: We summarize the results for the measurement of the residuals of the sensors under test for each ladder (L3 to L6) and side $(r-\phi, z)$ of the SVD sector. Here $\sigma$ is the width of the Gaussian function used to fit the residual distribution. The width of the residual, corrected by the uncertainty on the track position extrapolation on the sensor, $\sigma_{\text {track }}$, is also our measured intrinsic hit resolution, being the contribution to the residual due to multiple scattering negligible in our setup. As a comparison with the expected resolution for perpendicular tracks, the last column reports the resolution $p /(2 \sqrt{12})$ expected for a digital readout.

planes. Badly fitted tracks are removed from the analysis by rejecting tracks with a $\chi^{2} / n d o f>3.3$. From the simulation the uncertainty on the extrapolation of the reconstructed track position on the SVD sensor planes, $\sigma_{\text {track }}$, is of the order of $\approx 6-7 \mu \mathrm{m}$ for the $r-\phi$-direction and $\approx 8 \mu \mathrm{m}$ for the $z$-direction, respectively.

An example for the measured residuals is shown in Figure 4 and the numerical results are summarized in Table 1. Since the effect of multiple scattering is expected to be negligible in our setup, we estimate the resolution from the width of the residual distribution corrected by the error on the position of the extrapolated track on the sensor. For perpendicular tracks we expect a digital resolution $p /(2 \sqrt{12})$, where $p$ is the read out pitch of the sensor, the factor of 2 takes into account that the SVD sensors have a floating strip between two readout strips. We find that the measured resolutions are in good agreement with the expectation.
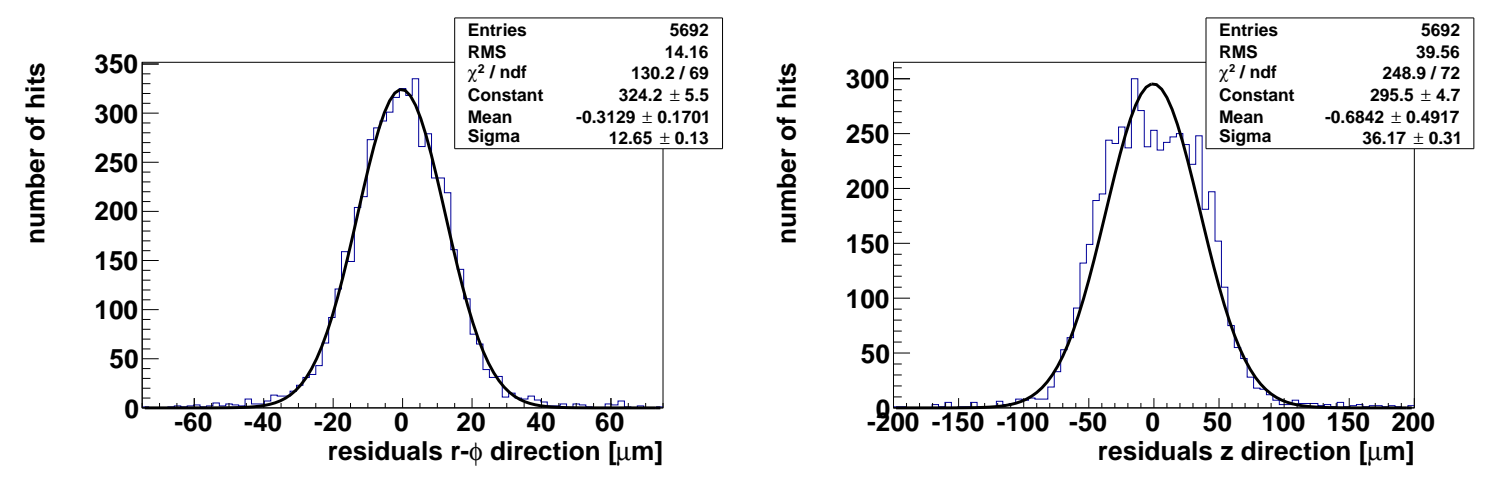

Figure 4: Residuals for the 3rd SVD layer of the sector under test (L5). The line represents a fit of a Gaussian to the residual.

\section{Summary}

A sector of the Silicon Vertex Detector and the Pixel detector of the Belle II experiment have been tested in a test beam at DESY Hamburg (Germany). The efficiencies for finding a hit have 
been estimated to be larger than 99\% for all the layers of the SVD, and the measured resolutions are consistent with the expectations.

\section{Acknowledgement}

The measurements leading to these results have been performed at the Test Beam Facility at DESY Hamburg (Germany), a member of the Helmholtz Association (HGF).

\section{References}

[1] T. Abe et al., Belle II Technical Design Report, [arXiv:1011.0352]

[2] Boqun Wang, The Belle II Experiment and SuperKEKB Upgrade, [arXiv:1511.09434]

[3] J. Schieck et al., DEPFET Pixels as a Vertex Detector for the Belle II Experiment, [arXiv:1304.0870v1]

[4] K. Adamczyk et al., The silicon vertex detector of the Belle II experiment, Nuclear Instruments and Methods in Physics Research, Section A: Accelerators, Spectrometers, Detectors and Associated Equipment, Volume 824, 11 July 2016, Pages 406-410

[5] S. Yamada, Data Acquisition System for the Belle II Experiment, IEEE Trans. Nucl. Sci. 62 number 3 pages 1175-1180(2015)

[6] H. Jansen et al., Performance of the EUDET-type beam telescopes, [arXiv:1603.09669]

[7] T. Schlüter, Vertexing and Tracking Software at Belle II, PoS Vertex2014 p. 039 (2014)

[8] Git repository for the used software: https://bitbucket.org/BenjaminSchwenker/tbsw.git

[9] Benjamin Schwenker, Development and validation of a model for the response of the Belle II vertex detector, https://ediss.uni-goettingen.de/handle/11858/00-1735-0000-0023-992D-9

[10] iLC Soft web page: http://ilcsoft.desy.de/portal 